\title{
NOTA CIENTIFICA
}

\section{Registro adicional de Langea euprosopides (Coleoptera: Cicindelidae) para el Perú}

\section{Additional record of Langea euprosopides (Coleoptera: Cicindelidae) for Peru}

\author{
Abdhiel Arnaldo Bustamante-Navarrete \\ Colección Entomológica de la Universidad del Cusco (CEUC), Facultad de Ciencias, Universidad Nacional San Antonio Abad del Cusco (UNSAAC), Pabellón \\ C, Ambiente 333, Ciudad Universitaria de Perayoc, Avenida de la Cultura 733, Cusco, Perú. \\ Dirección para correspondencia: Av. Manco Inca 319, Wanchaq, Cusco, Cusco, Perú. \\ Email Abdhiel A. Bustamante-Navarrete: abdhiel77@gmail.com \\ ORCID: http://orcid.org/0000-0001-8120-1274
}

\section{Resumen}

Se registra un nuevo espécimen de Langea euprosopides W. Horn, 1901 en la región Cusco (Perú). El género Langea W. Horn contiene tres especies. Debido a la falta de observaciones de miembros de este género en su ambiente natural, se desconocen sus hábitos, ciclo biológico y sus estadios de ciclo de vida. El género es aparentemente endémico del Perú. Se presentan algunos datos sobre el posible sustrato de captura y un mapa de distribución de la especie en el Perú.

Palabras clave: Coleóptera; Cicindelidae; Cicindelinae; Langea; taxonomía; sistemática; escarabajos tigre.

\section{Abstract}

A new specimen of Langea euprosopides W. Horn, 1901 is recorded from the Cusco region of Perú. The genus Langea W. Horn presently contains three species. Due to a lack of observations of members of this genus in the wild, its habits, natural history and life cycle stages are unknown. The genus is apparently endemic to Perú. Some data on the likely capture substrate and a distribution map of the species in Perú are presented.

Keywords: Coleoptera; Cicindelidae; Cicindelinae; Langea; taxonomy; systematics; tiger beetles.

\section{Citación:}

Bustamante-Navarrete A.A. 2016. Registro adicional de Langea euprosopides (Coleoptera: Cicindelidae) para el Perú. Revista peruana de biología 23(3): 311 - 314 (Diciembre 2016). doi: http://dx.doi.org/10.15381/ rpb.v23i3.12867

\section{Presentado: $\quad 24 / 02 / 2016$ \\ Aceptado: $\quad 22 / 09 / 2016$}

Publicado online: $20 / 12 / 2016$
Fuentes de financiamiento: el presente trabajo ha sido realizado gracias al proyecto Canon "Diversidad y distribución geográfica de la fauna regional del Cusco". 


\section{Introducción}

El género Langea fue establecido por W. Horn (1901) para la especie Langea euprosopides W. Horn, 1901. Posteriormente el mismo autor describió a Langea fleutiauxi (1915). Rivalier (1971) efectuó una revisión de la tribu Cicindelini, donde debido a la ausencia de material fue incapaz de ubicar a Langea en la nueva subtribu Iresina.

El género Iresia Dejean 1831 tiene especies muy parecidas a las de Langea, pero esta es distinguible por su labrum con 7 dientes y con 6 setas, el primer segmento del palpo labial expandido y en las hembras por la muesca del quinto segmento abdominal bajo el cuarto segmento. El género Langea es extremadamente raro en las colecciones, no se sabe mucho acerca de sus hábitos y hábitats, las larvas son desconocidas y se asume que los adultos son arbóreos, como es el caso del género hermano Iresia (Sumlin 1993).

La tercera especie del género, L. mellicollis, fue descrita en la revisión del género efectuada por Sumlin (1993), donde además efectuó el análisis de L. euprosopides, especie descrita de la localidad de Chancamayo, Cuzco (=Cusco), con registros adicionales en el Valle de Kosñipata (provincia de Paucartambo, región Cusco) y la localidad de Tingo María (región Huánuco). Sumlin encontró diferencias entre el holotipo hembra proveniente de Chancamayo (Fig. 3) y el espécimen hembra de Tingo María (Fig. 4) principalmente en la forma del labrum (Figs. 5 y 7), la rugosidad profunda (ausente en la especie de Tingo María) y el patrón de manchas marcadamente diferente. Debido a la ausencia de material, Sumlin ubico al ejemplar atípico de Huánuco tentativamente como L. euprosopides hasta reunir el material suficiente para determinar el estatus de este morfotipo.

En la Colección Entomológica de la Facultad de Ciencias de la Universidad Nacional San Antonio Abad del Cusco (CEUC) se ubicó un ejemplar de Langea euprosopides dentro del material de cicindélidae (Figs. 1 y 2). Este ejemplar presenta afinidad con los dos morfotipos anteriormente reportados, la conformación de las manchas elitrales es semejante al ejemplar de Huánuco (salvo la mancha humeral que es menos conspicua) y el labrum es semejante al ejemplar tipo (Chancamayo, Cusco). La localidad de colecta de esta nueva cita se ubica en la localidad de San Pedro ubicada en la vía de acceso al Valle de Kosñipata, el punto de colecta del otro registro conocido de L. euprosopides (Fig. 6).

\section{Taxonomía}

Orden Coleoptera Linnaeus 1758

Familia Cicindelidae Latreille 1802

Subfamilia Cicindelinae Csiki 1906

Genero Langea W. Horn 1901

\section{Especie Langea euprosopides W. Horn 1901}

Material examinado: 01 ejemplar: PERU; San Pedro, Kosñipata, Paucartambo, 1500 m [msnm], 07-nov-99 [1999]; sp071108IF03, (fruta); código de colección ColCic0003.
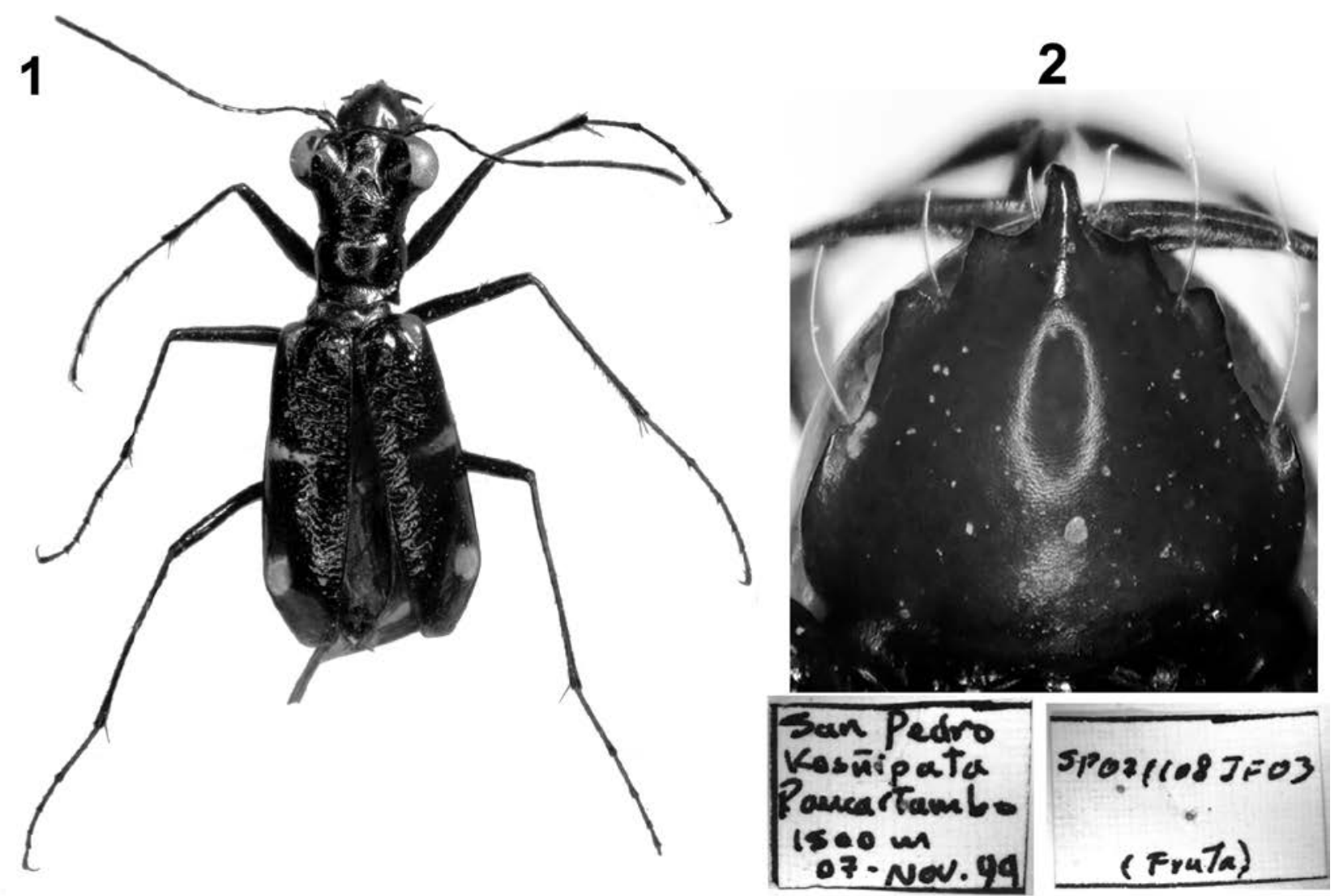

Figuras 1 - 2. (1) Habitus del ejemplar hembra de San Pedro (Cusco); (2): Detalle del labrum del ejemplar hembra de San Pedro (Cusco); 

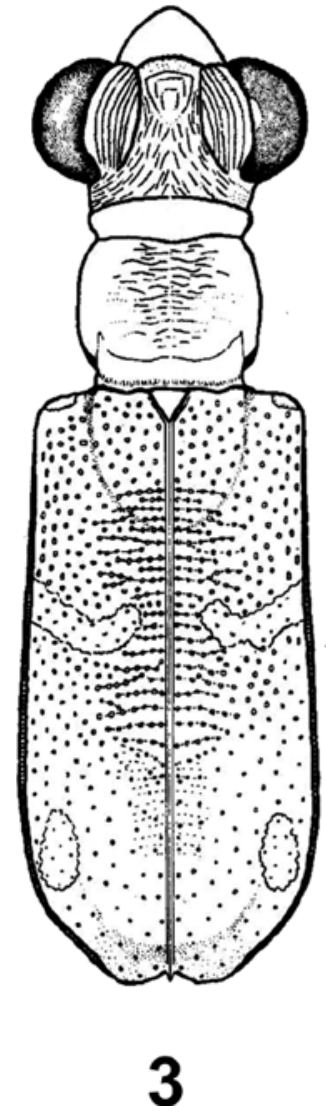

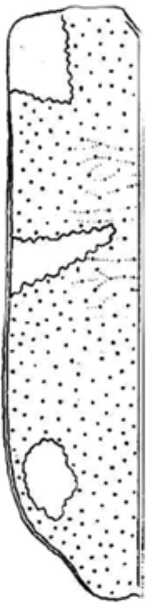

4

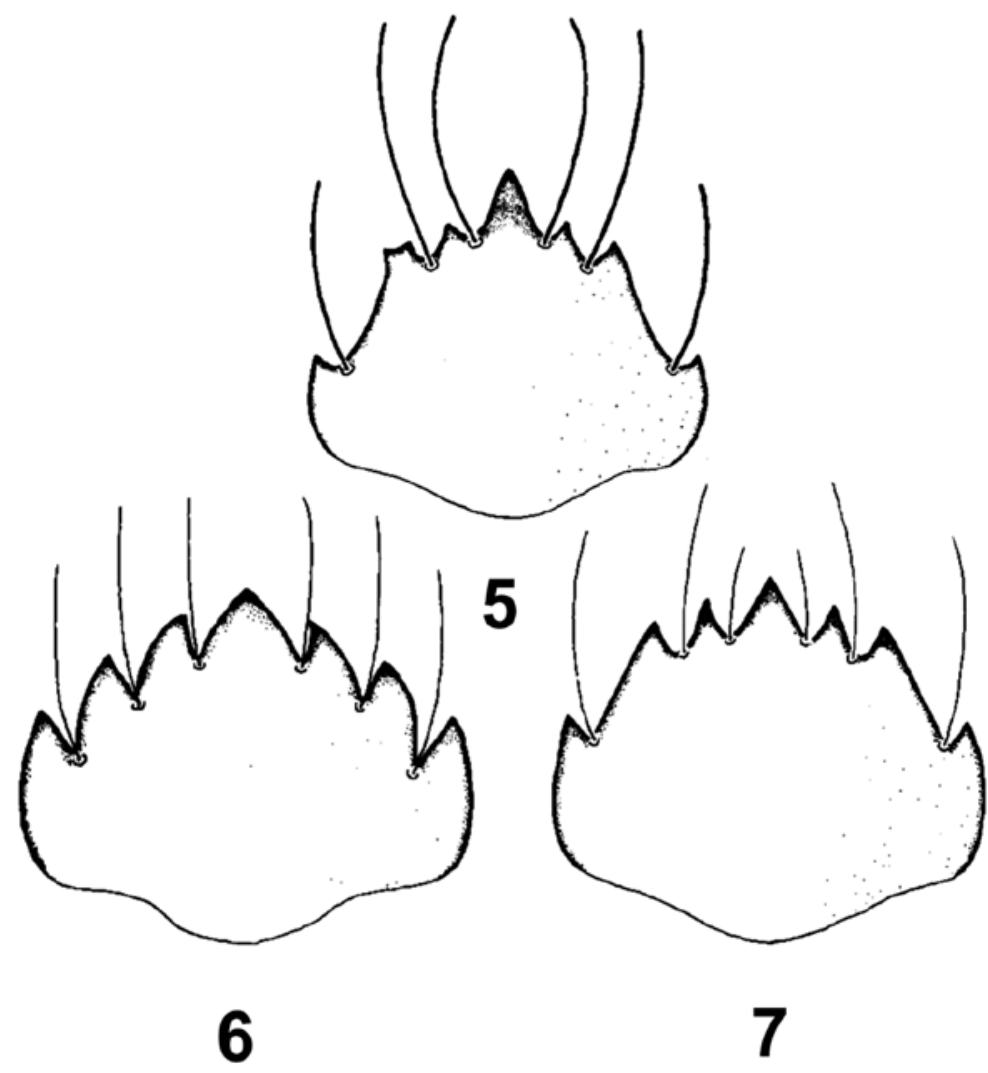

Figuras 3 - 7. (3): Habitus del holotipo hembra (Chancamayo, Cusco); (4): Detalle del élitro del ejemplar hembra de Huánuco; (5): Detalle del labrum del holotipo hembra (Chancamayo, Cusco); (6): Detalle del labrum del ejemplar macho de Kosñipata (Cusco); (7): Detalle del labrum del ejemplar hembra de Tingo María (Huánuco). Figuras 03, 04, 05, 06, 07 redibujados de Sumlin (1993).

Depositado en la Colección Entomológica de la Universidad Nacional San Antonio Abad del Cusco (CEUC). Datos adicionales de colecta: 19L 2239668555375.

Registros conocidos: Holotipo, hembra, Lange, Perú, Chanchamayo (depositado en Deutsches Entomologisches Institut, Eberswalde-Finow, Alemania); 01 ejemplar macho, Depto. Cuzco (=Cusco), Cosnipata-Ebene (=Kosñipata), 1000 m, 22-29-xii-1900 (depositado en Ronald L. Huber Collection, Bloomington, Minnesota); 01 ejemplar hembra, depto. Huánuco, Tingo María, 27-x-1937 (depositado en William D. Sumlin Collection, San Antonio, Texas).

Localidad tipo: Perú: Depto. Cuzco (=Cusco), Chancamayo.

Comentarios: Langea euprosopides es considerado como endémico del Perú (Cassola \& Pearson 2001),

\section{Discusión}

En la etiqueta del ejemplar colectado se registra el dato "fruta" que podría indicar el atrayente de una trampa o el sustrato de la colecta, si bien la especie es registrada como de hábitos diurnos y costumbres arborícolas (Pearson 1994), debido a su afinidad con el género Iresia. La escasez de material dificulta de momento el estudio de la biología de esta especie y en general de todo el género. Las localidades de colecta de esta especie y las otras dos del género parecieran apuntar a una distribución restringida al sur oriente del Perú (selva de las Regiones Cusco y Madre de

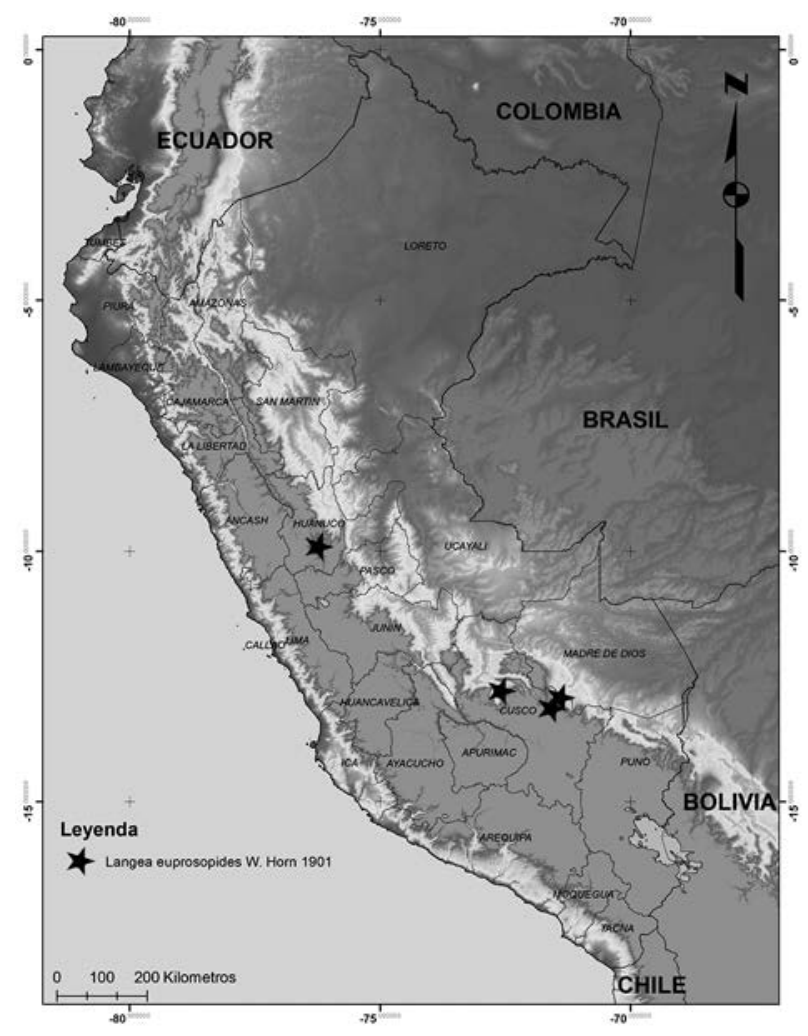

Figura 8. Distribución actual de Langea euprosopides W. Horn 1901 en el Perú. 
Dios), sin embargo el registro en Tingo María podría indicar métodos de colectas insuficientes o inadecuados en la región central del Perú.

\section{Agradecimientos}

A Ronald Huber, editor de la revista CICINDELA, por proporcionar la revisión del genero Langea. Al Dr. Erick Yábar Landa, responsable del proyecto Canon "Diversidad y distribución geográfica de la fauna regional del Cusco".

\section{Literatura citada}

Cassola F. \& D.L. Pearson. 2001. Neotropical tiger beetles (Coleoptera; Cicindelidae): checklist and biogeography. Biota Colombiana. 2(1):3-24.

Horn W. 1901. De 7 novis cicindelis. Deutsche Entomologische Zeitschrift, 353-358. Descarga disponible en: http://biodiversitylibrary.org/page/33086831

Horn W. 1915. Langea fleutiauxi nov spec. (Col., Cic.). Entomologische Mitteilungen, 4:206-206. Descarga disponible en: http://biodiversitylibrary.org/page/10021942

Pearson D.L. 1994. "Escarabajos tigre" del Perú (Coleoptera: Cicindelidae). Lista preliminar de especies y clave para géneros. Revista Peruana de Entomología 36:55-58.

Rivalier E. 1971. Remarques sur la tribu des Cicindelini (Col. Cicindelidae) et sa subdivisión en sous-tribus. Nouvelle Revue D'Entomologie. 1:135-143.

Sumlin W.D. 1993. Studies on the Neotropical Cicindelidae III: a review of the genus Langea (Coleoptera). Cicindela 25:1-12. 